\title{
How to Predict the Product Reliability Confidently and Fast with a Minimum Number of Samples in the Wöhler Test
}

\author{
Jens Mischko ${ }^{1(\bowtie)}$, Stefan Einbock ${ }^{1}$, and Rainer Wagener ${ }^{2}$ \\ 1 Robert Bosch GmbH, Schwieberdingen, Germany \\ Jens-Frederik. Mischko@de. bosch.com \\ 2 Fraunhoferinstitut LBF, Darmstadt, Germany
}

\begin{abstract}
To accurately estimate and predict the (product) lifetime, a large sample size is mandatory, especially for new and unknown materials. The realization of such a sample size is rarely feasible for reasons of cost and capacity. The prior knowledge must be systematically and consistently used to be able to predict the lifetime accurately. By using the example of Wöhler test, it will be shown that the lifetime prediction with a minimum number of specimen and test time can be successful, when taking the prior knowledge into account.
\end{abstract}

Keywords: Increasing efficiency $\cdot$ Wöhler test $\cdot$ Lifetime model • Prior knowledge $\cdot$ Test planning

\section{Introduction}

The reliable design of components or design elements is an essential part of the development areas in the product development process. This is particularly the case if oversizing, e.g. lightweight engineering, must be avoided. In order to be able to accurately estimate the strength, lifetime models are used. Lifetime models show the correlation between the stress or load and the lifetime of a failure mechanism. The challenge for test engineers is to determine the parameters of the lifetime models as accurately as possible based on lifetime tests in order to obtain the best possible estimation of the predicted lifetime. However, this can only be achieved with a very large sample size, as the test results are statistically distributed. In contrast, shorter development times and increasing competitive pressure lead to a lack of time and costs to determine the parameters of the lifetime models in a confident manner.

\section{Results}

This paper aims to show a method that can efficiently determine the parameters of lifetime models. By estimating the parameters based on prior knowledge, the

(C) The Author(s) 2021

P. F. Pelz and P. Groche (Eds.): ICUME 2021, LNME, pp. 115-128, 2021.

https://doi.org/10.1007/978-3-030-77256-7_11 
test conduction can be optimised. First results show that the parameters can be reasonably estimated even with a very small sample size of $n=5$ samples.

A high accurate knowledge regarding the parameters for lifetime models, ensure better results of the method. The prior knowledge can for example be determined through prior tests, based on the results of large statistical data collection or derived from mathematical models of guidelines by starting from chemical and physical material properties. However, the method can also be successfully applied to new or unknown materials or material qualities where only a limited amount of prior knowledge is available, e.g. in the form of previous test results.

The increased efficiency of this method is demonstrated by using a real Wöhler test. It turns out that a good estimation of the slope $k$ in particular leads to a good estimation of the Wöhler curve. In the example, the number of samples can be reduced by $75 \%$ from $n=20$ to $n=5$.

In order to examine the validity of the method, further statistical investigations must be carried out. Furthermore, the extension of the method to other lifetime models is to be examined.

\section{General Structure of Lifetime Models}

In general, lifetime models specify the correlation between the sustainable stress or load and the expected lifetime. This correlation is described by a mathematical model. Characteristic for lifetime models is that a lower lifetime can be expected at a higher load. However, there are limits to this correlation in most cases. The load is limited by the change of failure mechanism upwards and downwards. On the one hand, a static limit value can be reached upwards and on the other hand, no degradation occurs if the value is below a certain threshold value.

Examples of lifetime models are the Wöhler model or the Arrhenius model. The Wöhler model describes the fatigue of varying cyclic mechanical loads on the lifetime of materials. Upwards, the model is limited by the static failure of plasticisation. In contrast, the Arrhenius model describes the dependence of the reaction rate of a chemical reaction with the absolute temperature. A prerequisite for the reaction is that the specific activation energy is exceeded [1]. Table 1 shows both models. Further lifetime models refer to [2] for example.

Table 1. Overview of different lifetime models

\begin{tabular}{l|l}
\hline Name & Model \\
\hline Wöhler & $N=N_{A} \cdot\left(\frac{S}{S_{A}}\right)^{-k}$ \\
\hline Arrhenius & $t=t_{0} \cdot \exp \left(\frac{E_{a}}{k \cdot T}\right)$ \\
\hline
\end{tabular}


A characteristic feature of lifetime models is that they usually only provide a simple, linear relationship between the applied load and the expected lifetime. Mostly, the individual models differ only in the scaling of the axes. The Wöhler curve for example uses a double logarithmic scaling and results in a linear decrease of the high cycle fatigue.

\section{Proposal for an Efficient Test Procedure for the Determination of Wöhler Curves}

In this chapter, a method will be introduced to conduct fast and efficient lifetime tests. The focus of this method will be on conducting Wöhler test. A transfer to other lifetime models is possible due to the similarities between the lifetime models as described in Sect. 3 .

Efficient Wöhler tests are limited to the high cycle fatigue, since a discrete value is determined in the experiments. The aim is to define a procedure in which the Wöhler curve can be determined efficiently with a sample size of $n=5$ test specimens.

To make this possible, research on the test conduction of Wöhler experiments will first be analysed and the results will be derived from it. In addition, prior knowledge will be used for tests with such a small sample size. Due to the small sample size, the test will also be conducted according to the pearl chain method, where the experiment with one sample is carried out at different load levels.

\subsection{Research on the Test Conduction of Wöhler Tests}

Essential investigations on Wöhler experiments were presented in MüLLER [3]. With the help of Monte Carlo simulations, Wöhler tests were simulated and conclusions were obtained. The following descriptions are based on this work results.

The mean load level of the Wöhler curve at a specific number of load cycles can be estimated unbiased. The greater the spread of the number of load cycles, the fewer samples have to be rejected due to an increasing slope. The higher the spread and the smaller the scatter of the Wöhler curve, the better the estimated accuracy of the Wöhler curve.

The situation is similar when estimating the slope based on test results. The slope is estimated independently of the predetermined slope. Small spreads $N_{\max } / N_{\min }<10$ partly lead to the rejection of the estimation, since a increasing Wöhler curve is predicted. The best prediction is achieved when the test results are distributed over the entire range of the high cycle fatigue. The smaller the scatter of the Wöhler curve, the better the slope can be estimated.

An estimation for the scatter is asymptotically unbiased. With a very small sample size, e.g. $n<7$, the scatter minor underestimated. The estimation is independent of the spread, the slope and the scatter range. The uncertainty of the estimated scatter range is too large for a sample size of $n \leq 5$. Therefore, the parameter cannot be reliably estimated with this method. 
According to an efficient test conduction the following aspects should therefore be noted and implemented:

- The mean load level and the slope of the Wöhler curve can be estimated without bias.

- A wide spread $N_{\max } / N_{\min }>10$ is to be aimed for a good estimation of the slope.

- The scatter range $T_{N}=N_{90 \%} / N_{10 \%}$ of the Wöhler curve cannot be reliably estimated with a sample size of $n=5$.

\subsection{Prior Knowledge of the Wöhler Curve Parameters}

Prior knowledge of the Wöhler curve parameters is required for an efficient test conduction in determining the Wöhler curve. The prior knowledge can be obtained from different sources. The following section will present some methods which provide substantial elements for this prior knowledge.

In general, an estimate of the characteristic values can always be made from previous tests results. Alternatively, similar tests can be researched in databases and these values can be used as prior knowledge.

The fatigue limit or mean load can be calculated using mathematical estimations. A well-known example for the calculation of the fatigue limit depending on various influencing parameters, such as the mean load, the size influence, the notch condition or surface influences can be found in the FKM guideline [4]. The fatigue limit is estimated on the basis of easily determined material properties. Calculations can also be conducted using the finite element method.

Calculated procedures for determining the slope of the Wöhler curve can be found, for example, in [5,7]. Proposals for an estimated slope can also be found in the FKM guideline [4]. Another possibility for predicting the slope is the concept of the standardised Wöhler curve, as described in [6].

With standardised Wöhler curves, the scatter range of the Wöhler curve can also be reliably predicted. Evaluations of the scatter ranges of Wöhler curves were published in detail in ADENSTEDT [8]. These results can be used for efficient test conduction.

A suggestion for the parameter of the Wöhler curve slope $k$ can be taken from the following Table 2 and for the scatter range from Table 3. If no scatter ranges are known, a scatter range in the direction of load cycles of $T_{N} \approx 3.25$ can be assumed according to $[8,9]$.

Table 2. Proposal for the slope $k$ of steel [10]

\begin{tabular}{l|l|l}
\hline Notched & Welded & Not welded \\
\hline Not & & $k=15$ \\
\hline Mildly & $k=3 \ldots 5$ & $k=5$ \\
\hline Sharply & & $k=3$ \\
\hline
\end{tabular}


Table 3. Proposal for the scatter range $T_{S}$ of steel $[6,8]$

\begin{tabular}{l|l|l}
\hline Notched & Welded & Not welded \\
\hline Not & & $T_{S}=1.10 \ldots 1.15$ \\
\hline Mildly & $T_{S}=1.25 \ldots 1.45$ & $T_{S}=1.20 \ldots 1.30$ \\
\hline Sharply & & $T_{S}=1.25 \ldots 1.30$ \\
\hline
\end{tabular}

\subsection{Derivation of an Efficient Test Conduction}

An efficient method for conducting Wöhler tests will be introduced, in this section. The results regarding the test conduction described in Sect. 4.1 and take prior knowledge into account as presented in Sect.4.2. The procedure is based on the following basic ideas:

- the test results should confirm the existing prior knowledge,

- prior knowledge is valid until it has been rejected by the experimental test results.

For this purpose, the high cycle fatigue area of the Wöhler curve is divided into five sectors. In general, the high cycle fatigue area starts at $N=1 \cdot 10^{4}$ numbers of load cycles [9] at the latest and ends at about $N=1 \cdot 10^{6}$ numbers of load cycles. The aim is to have a test result in each of the five sectors. In an experiment, the load $S$ must always be specified and the number of load cycles $N$ is the statistically dependent parameter. Therefore, it is not trivial to construct a test where the number of load cycles covers the specified range. The experimental results should ideally be conducted according to the order shown in Fig. 1. The order was chosen considering the following aspects:

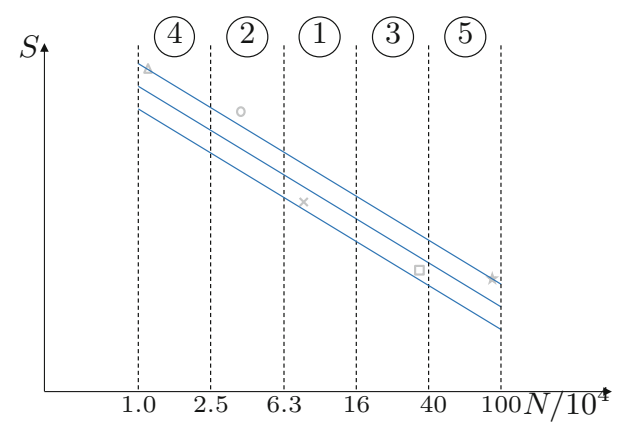

Fig. 1. Dividing the high cycle fatigue area from $N=1 \cdot 10^{4}$ to $N=1 \cdot 10^{6}$ into five sections

1. The test point is located in the centre of the high cycle fatigue area. This increases the probability that even if the Wöhler curve is poorly estimated, the test point will still be within the high cycle fatigue area. 
2. The second test level is above the load level of the first and has a shorter test time than the first test point. This test should be in the second of five sections and thus continue to be as safe as possible in the area of fatigue strength.

3. The third sample is in the fourth section and thus further within a safe section of the high cycle fatigue area.

4. In order to achieve the largest possible spread of the Wöhler curve, the fourth test point is located in the first section and thus as close as possible to the low cycle fatigue area. This sample is chosen in a way that it has a short test time.

5. The fifth sample is close to the transition area, to further maximise the spread. As this is the test point with the longest test time, it should also be estimated best. Therefore, the fifth sample is conducted at the end of the test series.

After each of the conducted tests it will be assessed, whether the result of the experiment applies to the prior knowledge. If exactly $n=1$ test result is known, then, as shown in the Fig. 2, the test result is compared to the Wöhler curve by checking whether the result lies within or outside the $95 \%$ interval of the Wöhler curve:

$$
N_{1} \in\left(N_{1,2.5 \%}, N_{1,97.5 \%}\right)
$$

If the result is within the given range, the Wöhler curve from the prior knowledge is assumed. The second test level is determined with the prior knowledge. However, if the test result is outside the defined limits, the Wöhler curve is moved parallel through the test result and the corrected prior knowledge is used to determine the next test level, see Fig. 2.

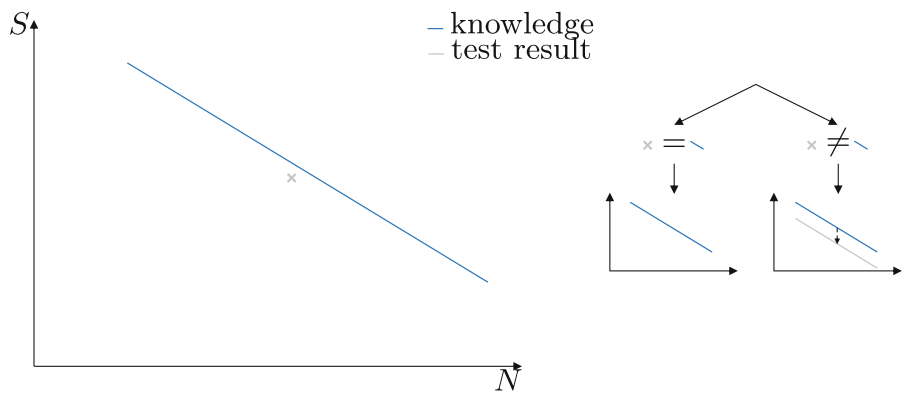

Fig. 2. Testing strategy for $n=1$ samples

If at least two tests have been conducted at two different load levels, then both the slope $k$ and the mean load level from the test results $\bar{S}$ can be compared with the prior knowledge. The procedure is shown in Fig. 3: 


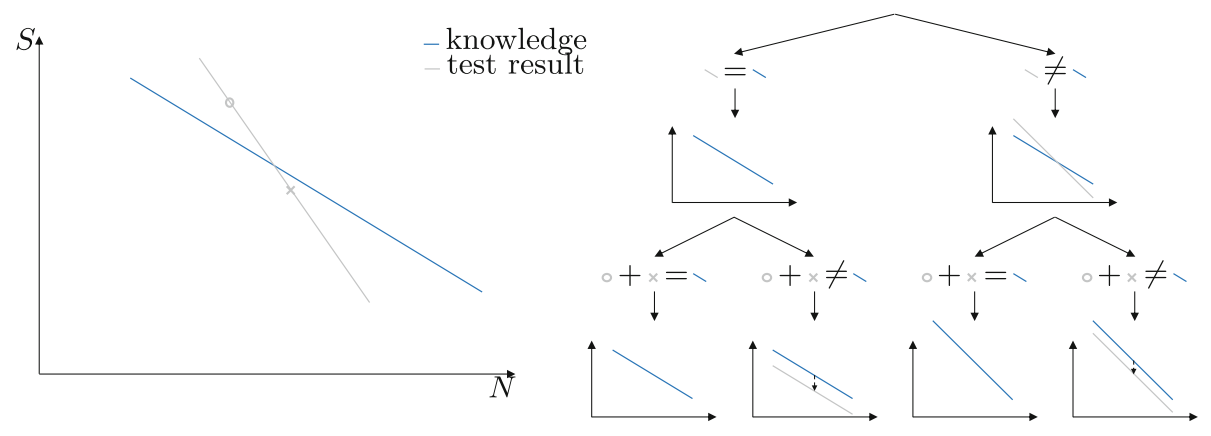

Fig. 3. Testing strategy for $n>1$ samples

1. Check the slope from the prior knowledge $k_{0}$ with the slope $k$ determined from the test results.

2. Verify the mean load level from prior knowledge $S_{0}$ with the mean load level $\bar{S}$ determined from the test results. Depending on the prior test decision, the slope from the prior knowledge $k_{0}$ or the slope $k$ determined from the regression calculation is chosen for the verification.

Since there are now at least two samples, the parameters determined from the experimental results and the prior knowledge can be tested using a twosided one-sample $t$-test. Within the method it is specified that the results are conducted at the significance level of $\alpha=5 \%$. The sample test is divided into three steps [11]:

1. Formulate null hypothesis:

$$
\begin{aligned}
& H_{0}: \mu=\mu_{0} \\
& H_{1}: \mu \neq \mu_{0}
\end{aligned}
$$

2. Calculate test value:

$$
t=\sqrt{n} \cdot \frac{\bar{x}-\mu_{0}}{s}
$$

3. Test decision:

Rejection area for $H_{0}:\left(-\infty,-t_{1-\frac{\alpha}{2} ; n-1}\right) \cup\left(t_{1-\frac{\alpha}{2} ; n-1}, \infty\right)$

In the first sample test, the slope is tested. Therfore, the slope $k$ determined from the regression calculation is tested against the slope from the prior knowledge $k_{0}$. In order to be able to conduct the sample test, a scatter of the slope $s_{k}$ is required. This depends on the following factors:

- Sample size $n$

- Spread $N_{\max } / N_{\min }$

- Slope $k$

- Scatter range in direction of the load cycles $s_{N}=\frac{\log \left(T_{N}\right)}{2.56}$

Monte Carlo simulations with different parameter variations were conducted to determine the scatter of the slope as a function of the mentioned parameters. The 
general relationship for the scattering parameter of the slope $s_{k}$ was determined in Eq. 1. This parameter depends on the slope $k$ itself, the scatter in the direction of load cycles $s_{N}$ and a further scatter parameter $s\left(n, N_{\max } / N_{\min }\right)$ :

$$
s_{k}=k \cdot \frac{\frac{1+s_{N} \cdot s\left(n, N_{\max } / N_{\min }\right)}{1-s_{N} \cdot s\left(n, N_{\max } / N_{\min }\right)}-1}{\frac{1+s_{N} \cdot s\left(n, N_{\max } / N_{\min }\right)}{1-s_{N} \cdot s\left(n, N_{\max } / N_{\min }\right)}+1}
$$

The scattering parameter $s\left(n, N_{\max } / N_{\min }\right)$ can be tabulated or calculated using the relationship given in Eq. 2:

$$
s\left(n, N_{\max } / N_{\min }\right)=\frac{a(n)}{\ln \left(N_{\max } / N_{\min }\right)}
$$

The constant $a(n)$ is only dependent on the sample size $n$. The characteristic values for this parameter can be taken from the Table 4 .

Table 4. Characteristic values for the calculation of the parameter $s\left(n, N_{\max } / N_{\min }\right)$, see Eq. 2

\begin{tabular}{l|l|l|l|l|l|l}
\hline$n=$ & 2 & 3 & 4 & 5 & 7 & 10 \\
\hline$a(n)=$ & 3.256 & 3.255 & 3.089 & 2.911 & 2.610 & 2.282 \\
\hline
\end{tabular}

The mean load level $\bar{S}$ is to be tested with the mean load level from the prior knowledge $S_{0}$ in a second one-sample test. The load level is calculated depending on the determined slope from the first test decision, see Fig. 3. The individual test points are transformed via the slope $k$ to the level of the mean number of load cycles $N_{0}$ from the prior knowledge. The following characteristic values are then used for the test:

$$
\begin{aligned}
& \mu=\log (\bar{S})=\frac{1}{n} \sum_{i=1}^{n}\left(\log S_{i}\right) \\
& \mu_{0}=\log \left(S_{0}\right)
\end{aligned}
$$

Using prior knowledge, the scatter range in load direction $T_{S}$ is already defined. For the one sample test this can be used by the following calculation:

$$
s_{S}=\frac{\log \left(T_{S}\right)}{2.56}
$$

\section{Example of Increasing the Efficiency of Costs and Time When Conducting Wöhler Tests}

\subsection{Data Basis}

The procedure for efficient test conduction is demonstrated by using a concrete test result. The test and its results are shown in the Table 5 . 
Table 5. Results of a Wöhler test

\begin{tabular}{lll}
\hline$i$ & $S_{i}$ & $N_{i}$ \\
\hline 1 & 114 & $3.61 \cdot 10^{5}$ \\
2 & 128 & $1.23 \cdot 10^{5}$ \\
3 & 128 & $7.45 \cdot 10^{4}$ \\
4 & 114 & $4.73 \cdot 10^{5}$ \\
5 & 117 & $3.87 \cdot 10^{5}$ \\
6 & 133 & $1.75 \cdot 10^{4}$ \\
7 & 133 & $3.49 \cdot 10^{4}$ \\
8 & 133 & $3.76 \cdot 10^{4}$ \\
9 & 117 & $3.17 \cdot 10^{5}$ \\
10 & 107 & $5.13 \cdot 10^{5}$ \\
\hline
\end{tabular}

\begin{tabular}{lll}
\hline$i$ & $S_{i}$ & $N_{i}$ \\
\hline 11 & 117 & $3.41 \cdot 10^{5}$ \\
12 & 128 & $7.51 \cdot 10^{4}$ \\
13 & 101 & $4.29 \cdot 10^{5}$ \\
14 & 101 & $3.55 \cdot 10^{5}$ \\
15 & 101 & $6.86 \cdot 10^{5}$ \\
16 & 133 & $4.67 \cdot 10^{4}$ \\
17 & 128 & $1.28 \cdot 10^{5}$ \\
18 & 117 & $3.94 \cdot 10^{5}$ \\
19 & 101 & $1.98 \cdot 10^{6}$ \\
20 & 99 & $6.94 \cdot 10^{5}$ \\
\hline
\end{tabular}

A total of $n=20$ samples were conducted at seven test levels. All test results are shown normalised, where the value $S=100$ corresponds to the load level at $N_{0}=1 \cdot 10^{6}$ which in this case is $36 \%$ of the tensile strength $R_{m}$. The test series shown originates from a $K_{t}=1$ sample of a material. The evaluation of the entire test series from Table 5 resulted in the following:

$$
\begin{aligned}
k & =10 \\
T_{S} & =1.15 \\
S_{0}\left(N_{0}=1 \cdot 10^{6}\right) & =0.36 \cdot R_{m}=100
\end{aligned}
$$

\subsection{Evaluation Taking Prior Knowledge into Account}

In this test, a material with comparatively few preliminary tests had been examined. Characteristic strength values and Wöhler curve parameters can only be taken from the literature to a limited extent and not from standards. However, the following characteristic values could be derived from the existing preliminary tests:

$$
\begin{aligned}
k & =10 \\
T_{S} & =1.15 \\
S_{0}\left(N_{0}=1 \cdot 10^{6}\right) & =0.27 \cdot R_{m}=75
\end{aligned}
$$

Procedure for Evaluating the Test Results. The test results from Table 5 should be taken to apply the method described in Sect. 4.3. The following principle is applied in each case:

1. The next test level is calculated by using the prior knowledge or the prior knowledge corrected by the test results. 
2. The test level closest to the calculated test level is then selected.

3. If several tests were conducted at the test level, then the test result is taken into account in the order of the tests conducted. This also applies if a test level is selected several times.

4. If the second test level corresponds to the first test level or if no further test results are available, the test level that is second closest to the calculated test level is used as an exception.

In order to better classify the results, different variants were chosen for the evaluation of the test results. In the first part, the correct slope was used and the mean load level was varied. In a second evaluation, the correct mean load level was specified and the slopes varied.

\section{Specification of the Correct Slope and Variation of the Mean Load}

Level. The prior knowledge used for the tests is shown in Table6. For variant A, the mean load level was underestimated by $25 \%$ which corresponds to the estimation as it would have been conducted in the experiment, refer to Eq. 8. Variant $\mathrm{C}$ corresponds to the result as it turned out in the evaluation of all test results.

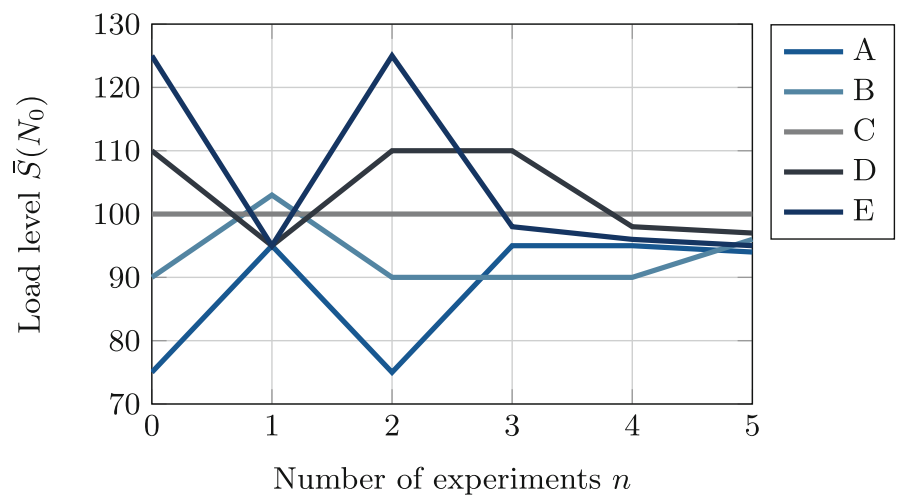

Fig. 4. Evaluated mean load level $\bar{S}$ at $N_{0}=1 \cdot 10^{6}$ for the prior knowledge $(n=0)$ and after $n=1 \ldots 5$ experiments. The prior knowledge for the variants is shown in Table 6 .

The results of these evaluations are shown in Fig. 4 and Fig. 5 as a function of the conducted samples. The slope was estimated correctly in each case and was not corrected in any of the cases. In contrast, the estimated mean load level was already corrected after the first test in all cases, with exception of variant C. After the tests were conducted, all test results were between $S=94$ and $S=100$. The deviation from the evaluation of all test results was therefore $<10 \%$.

Specification of the Correct Mean Load Level and Variation of the Slope. In the second evaluation of the experiment, the slope of the prior 


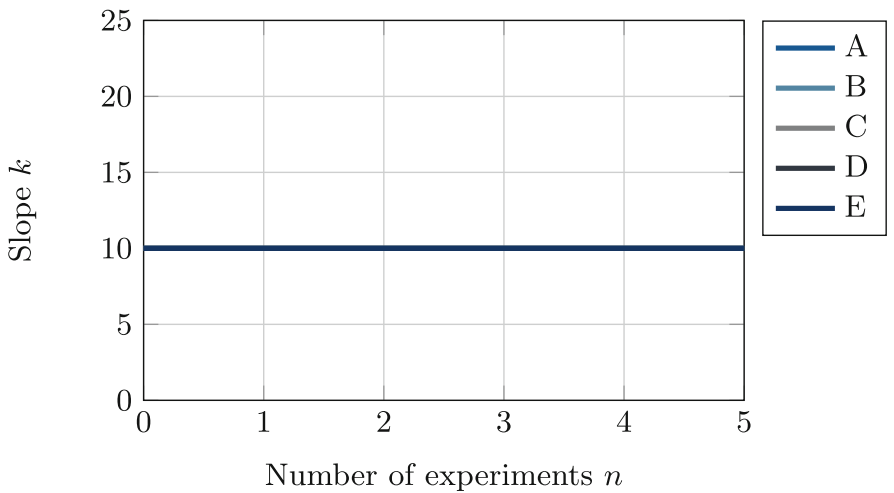

Fig. 5. Evaluated slope $k$ for the prior knowledge $(n=0)$ and after $n=1 \ldots 5$ experiments. The prior knowledge for the variants is shown in Table 6.

Table 6. Parameters for the different prior knowledge with a correct slope and a variation of the mean load level

\begin{tabular}{l|l|l|l|l}
\hline Variant & $T_{S}$ & $N_{0}$ & $S_{0}$ & $k_{0}$ \\
\hline $\mathrm{A}$ & 1.15 & $1 \cdot 10^{6}$ & 75 & 10 \\
\hline $\mathrm{B}$ & 1.15 & $1 \cdot 10^{6}$ & 90 & 10 \\
\hline $\mathrm{C}$ & 1.15 & $1 \cdot 10^{6}$ & 100 & 10 \\
\hline $\mathrm{D}$ & 1.15 & $1 \cdot 10^{6}$ & 110 & 10 \\
\hline $\mathrm{E}$ & 1.15 & $1 \cdot 10^{6}$ & 125 & 10 \\
\hline
\end{tabular}

knowledge is varied. The scatter range $T_{S}$ was chosen so that the scatter range in the load direction $T_{N}=T_{S}{ }^{k}$ was kept approximately constant. All variants are shown in Table 7 . With variant $\mathrm{c}$, the correct test result was given as prior knowledge as a reference.

Table 7. Parameters for the different prior knowledege with a correct mean load level and a variation of the slope

\begin{tabular}{l|l|l|l|l}
\hline Variant & $T_{S}$ & $N_{0}$ & $S_{0}$ & $k_{0}$ \\
\hline $\mathrm{a}$ & 1.30 & $1 \cdot 10^{6}$ & 100 & 5 \\
\hline $\mathrm{b}$ & 1.20 & $1 \cdot 10^{6}$ & 100 & 8 \\
\hline $\mathrm{c}$ & 1.15 & $1 \cdot 10^{6}$ & 100 & 10 \\
\hline $\mathrm{d}$ & 1.10 & $1 \cdot 10^{6}$ & 100 & 15 \\
\hline
\end{tabular}


The results are shown in Fig. 6 and Fig. 7. The slope was only corrected respectively after the fourth and fifth tests. For variants a and b, each of which underestimated the slope in prior knowledge, the slope was estimated too large in after conducting $n=5$ samples. The mean load level at the reference load cycle number of $N_{0}=1 \cdot 10^{6}$ was estimated too high. In contrast to that, due to the slope, the number of load cycle numbers for $N=1 \cdot 10^{4}$ was estimated too low. The two variants $\mathrm{c}$ and $\mathrm{d}$ on the other hand returned the correct results for both the slope and the mean load level.

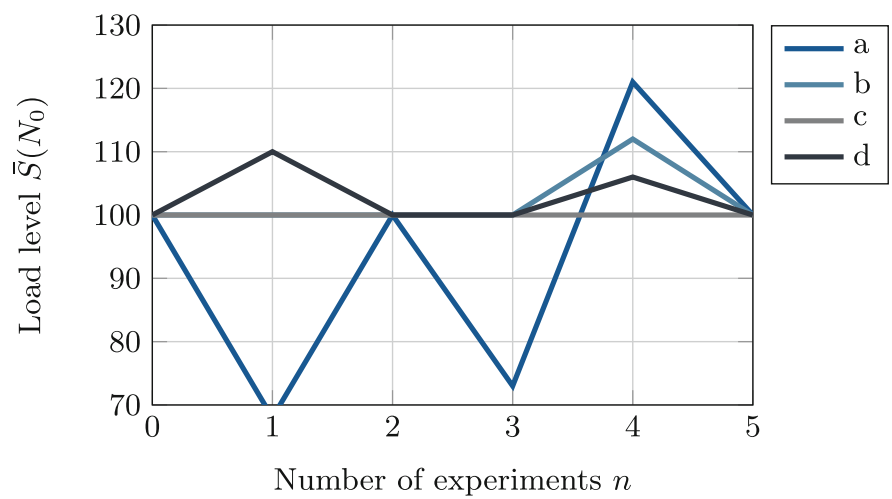

Fig. 6. Evaluated mean load level $\bar{S}$ at $N_{0}=1 \cdot 10^{6}$ for the prior knowledge $(n=0)$ and after $n=1 \ldots 5$ experiments. The prior knowledge for the variants is shown in Table 7 .

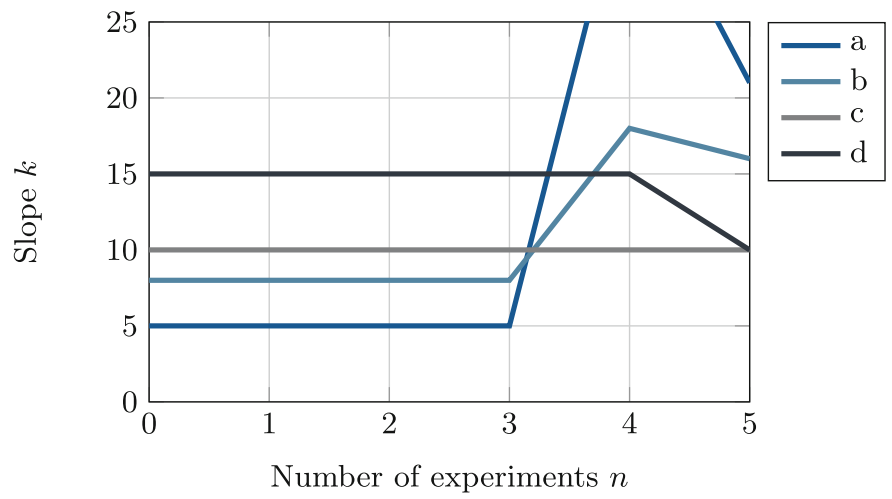

Fig. 7. Evaluated slope $k$ for the prior knowledge $(n=0)$ and after $n=1 \ldots 5$ experiments. The prior knowledge for the variants is shown in Table 7. 


\subsection{Concluions}

Using this example, good results can already be achieved for the experiment with a reduced sample size. Even with incorrectly assumed prior knowledge, it was possible to correct this circumstance and reliable results were already achieved after $n=5$ samples, which came close to the test results with $n=20$ samples. It is obvious that the mean load level is corrected much earlier with a wrong estimation than it is the case with a wrong estimated slope.

In this first example, the saving on specimens was $75 \%$. For the number of load cycles, the saving was even between $77 \%$ and $92 \%$. Good results could be achieved with the method when the slope was estimated properly. If the slope is estimated inaccurately, a larger sample size is needed.

The method focuses on the determination of the parameters for lifetime models. There are further uncertainties in the determination of product reliability that have not been addressed so far. This is the case when for example several failure mechanisms of a system are critical or influences of tolerances (geometry, surface quality, ...) are present.

\section{Outlook}

In a first example, it was shown that with the method for efficient test conduction taking prior knowledge into account, it is quite possible to significantly reduce the sample size. Good prior knowledge about the parameters of the lifetime model is an advantage. Further research will target the following three focus points:

- Further statistical studies on the method to demonstrate validity,

- Procedure for further validation of the lifetime model for $n>5$ samples,

- Transferability of the method to other failure mechanisms.

\section{References}

1. Bargel, H., Schulze, G.: Werkstoffkunde. Springer, Heidelberg (2013)

2. Jakob, F.: Nutzung von Vorkenntnissen und Raffungsmodellen für die Zuverlässigkeitsbestimmung. In: Berichte aus dem Institut für Maschinenelemente. PhD thesis (2017)

3. Müller, C.: Zur statistischen Auswertung experimenteller Wöhlerlinien. PhD thesis (2015)

4. FKM-Richtlinie Rechnerischer Festigkeitsnachweis für Maschinenbauteile aus Stahl, Eisenguss und Aluminiumwerkstoffen, 6. überarbeitete Auflage (2012)

5. Bergmann, J., Thumser, R.: Synthetische Wöhlerlinien für Eisenwerkstoffe. In: Forschungsbericht, p. 249 (1999)

6. Haibach, E.: Betriebsfestigkeit: Verfahren und Daten zur Bauteilberechnung. Springer, Berlin (2006)

7. Hück, M., Thrainer, L., Schütz, W.: Berechnungen von Wöhlerlinien aus Stahl. In: Bericht der Arbeitsgemeinschaft Betriebsfestigkeit, Stahlguß und Grauguß (1981)

8. Adenstedt, R.: Streuung der Schwingfestigkeit. PhD thesis (2001) 
9. DIN 50100: Schwingfestigkeitsversuch - Durchführung und Auswertung von zyklischen Versuchen mit konstanter Lastamplitude für metallische Werkstoffproben und Bauteile (2015)

10. Radaj, D., Vormwald, M.: Ermüdungsfestigkeit: Grundlagen für Ingenieure. Springer, Heidelberg (2010)

11. Einbock, S.: Statistik für Ingenieure und Naturwissenschaftler (mit Excel): endlich verständlich! (2018)

Open Access This chapter is licensed under the terms of the Creative Commons Attribution 4.0 International License (http://creativecommons.org/licenses/by/4.0/), which permits use, sharing, adaptation, distribution and reproduction in any medium or format, as long as you give appropriate credit to the original author(s) and the source, provide a link to the Creative Commons license and indicate if changes were made.

The images or other third party material in this chapter are included in the chapter's Creative Commons license, unless indicated otherwise in a credit line to the material. If material is not included in the chapter's Creative Commons license and your intended use is not permitted by statutory regulation or exceeds the permitted use, you will need to obtain permission directly from the copyright holder.

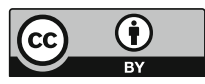

